\title{
RECENT DEVELOPMENTS IN THE THEORY OF LOCALLY CONVEX VECTOR SPACES
}

\author{
J. A. DIEUdONNÉ
}

1. Introduction. Although the theory of Banach spaces has been very popular among American mathematicians during the last twenty years, comparatively little attention seems to have been given, in this country, to its generalizations, except in the very last few years. With the exception of the outstanding work of G. W. Mackey $[47 ; 48]$, most contributions to the general theory of locally convex spaces have been made by European mathematicians. There may be some interest, therefore, in a survey in broad outline of the most recent advances in that field, some of which have not yet appeared in print.

The principal motivation behind the general theory is the same as that of Banach himself: namely, a search for general tools which might be applied successfully to functional analysis. Two different sectors contributed the main influences: the first originated in the work of G. Köthe, O. Toeplitz, and their students on sequence spaces $[32-46 ; 26 ; 50 ; 11]$, which began around 1934 and was partly related to the theory of functions of a complex variable [62]; many of the ideas which were to become fundamental in the later development of the general theory appeared there for the first time, and also a great wealth of illuminating examples and counter-examples. For unknown reasons, this remarkable pioneering work has to this day remained practically ignored in this country, in spite of its intrinsic importance and usefulness.

The other influence was exerted by the developments of the theory of integration, and chiefly through the efforts to free that theory from the shackles of the Caratheodory measure theory and turn it into a mere chapter of the general theory of topological vector spaces [6]. These efforts culminated in L. Schwartz's theory of distributions (1945), which could be expressed only in the language of locally convex vector spaces [56]; it turned out that for that theory, Banach spaces were an utterly inadequate tool, and the realization of that fact led to very active research on more general spaces, to which most of the results obtained in the last few years owe their origin.

This recent work has led in particular to a new classification and

An address delivered before the Chicago meeting of the Society on April 24, 1953 by invitation of the Committee to Select Hour Speakers for Western Sectional Meetings; received by the editors April 24, 1953. 
reëvaluation of the whole subject. Part of the interest which attached itself to the study of Banach spaces was certainly due to their particularly simple definition by means of a norm, and it is quite natural that this simple case should have been attacked first. But analysts are more interested in the properties of a space than in the way it is defined; hence the idea, first clearly formulated by L. Schwartz, of classifying topological vector spaces according to their behavior with regard to the validity of the main theorems of functional analysis. For instance, two of the most important of these, the Banach-Steinhaus theorem and the closed graph theorem, are valid in Banach spaces; it is quite natural to ask in what other spaces they are still true. This program has been very actively pushed forward within the last few years; its main outcome is that, surprisingly enough, practically no important property is really special to Banach spaces, although the latter still constitute a very useful technical tool in the study of the general theory.

2. The fundamental notions: I. Locally convex spaces (see [4]). We shall be exclusively concerned with vector spaces over the real field: the passage to complex spaces offers no difficulty. We shall assume that the definition and properties of convex sets are known. A convex set $A$ in a vector space $E$ is symmetric if $-A=A$; then $0 \in A$ if $A$ is not empty. A convex set $A$ is absorbing if for every $x \neq 0$ in $E$, there exists a number $\alpha \neq 0$ such that $\lambda x \in A$ for $|\lambda| \leqq \alpha$; this implies that $A$ generates $E$.

A locally convex space is a topological vector space in which there is a fundamental system of neighborhoods of 0 which are convex $[54 ; 63]$; these neighborhoods can always be supposed to be symmetric and absorbing. Conversely, if any filter base is given on a vector space $E$, and consists of convex, symmetric, and absorbing sets, then it defines one and only one topology on $E$ for which $x+y$ and $\lambda x$ are continuous functions of both their arguments.

A semi-norm on a vector space $E$ is a function $p(x)$ defined on $E$, such that $0 \leqq p(x)<+\infty$ for all $x \in E, p(\lambda x)=|\lambda| p(x)$, and $p(x+y)$ $\leqq p(x)+p(y)$; the sets defined by any of the relations $p(x)<\alpha$, $p(x) \leqq \alpha(\alpha>0)$ are convex, symmetric and absorbing; conversely, for every such set $A$, there exists one and only one semi-norm $p$ such that $A$ contains the set $p(x)<1$ and is contained in $p(x) \leqq 1$. From these remarks it follows that the topology of a locally convex space can also be defined by a family $\left(p_{\alpha}\right)$ of semi-norms, to which correspond the neighborhoods of 0 defined by $p_{\alpha}(x)<\lambda(\lambda>0)$; and conversely, such a family always defines a locally convex topology. This topology 
is Hausdorff if and only if, for every $x \neq 0$, there is an $\alpha$ such that $p_{\alpha}(x) \neq 0$; it is metrizable if the family $\left(p_{\alpha}\right)$ is denumerable.

3. The fundamental notions: II. Bounded sets (see [5]). The concept of bounded set is easily defined in a normed space: it is a set contained in some ball $\|x\| \leqq R$. To extend this notion when no metric is at hand, we may reformulate it as follows: $B$ is bounded if, given any ball $\|x\| \leqq r$, there exists a $\lambda>0$ such that $\lambda B$ is contained in that ball. If we say that a set $A$ absorbs a set $B$ if there exists $\lambda>0$ such that $\lambda B<A$, we can therefore say that a bounded set is one which is absorbed by every ball. Hence the general definition of a bounded set in a locally convex space $E$ : it is a set $B$ which is absorbed by every neighborhood of 0 in $E[31 ; 54]$. An equivalent definition is that every semi-norm which defines the topology of $E$ is bounded on $B$. If $B$ is bounded, so is $\mu B$ for any $\mu$; the convex hull of $B$ is bounded, as well as its closure. The union of a finite number of bounded sets is bounded; so is $A+B$, if both $A$ and $B$ are bounded. Precompact sets (in particularly Cauchy sequences) are bounded.

The notion of bounded set is not very important in normed spaces, because it is then equivalent to the notion of (arbitrary) subset of a ball; in other words, there is a fundamental system of bounded neighborhoods of 0 . This turns out to be exceptional among locally convex spaces: indeed, a Hausdorff locally convex space possesses bounded neighborhoods of 0 if and only if its topology can be defined by means of a norm [31].

On a locally convex space (as on any abelian topological group) there is a uniform structure determined by its topology, and such a space $E$ is said to be complete if every Cauchy filter (for that uniform structure) converges in $E$; for any Hausdorff locally convex space $E$, there is a well determined locally convex space $\widehat{E}$ which is complete and in which $E$ is dense (the completion of $E$ ). There are important locally convex vector spaces (for instance, all infinite-dimensional vector spaces with "weak" topologies; see $\$ 6$ below) which fail to be complete; but most spaces which occur in functional analysis have at least the weaker property that bounded closed sets are complete; they are called quasi-complete spaces. A still weaker property, which suffices for many applications, is that every Cauchy sequence is convergent, in which case the space is said to be semi-complete. The three notions coincide of course for metrizable spaces.

4. The 5 -topologies on the spaces $\mathcal{L}(E, F)[5 ; 7]$. The most important applications of locally convex spaces to functional analysis deal with linear operators, that is, linear mappings from a func- 
tional space $E$ into a functional space $F$, subject in general to conditions related to the topologies of $E$ and $F$. One is thus led, in particular, to study the set $\mathcal{L}(E, F)$ of all continuous linear mappings of a locally convex space $E$ into a locally convex space $F$. This is itself a vector space, and one of the main problems of the theory is to define and to study on $\mathcal{L}(E, F)$ topologies related in a natural way to those of $E$ and $F$. The known methods of defining topologies on functional spaces by conditions of "uniform smallness" on certain subsets [3] lead to the following tentative definition: for every subset $A$ of $E$ and every neighborhood $V$ of 0 in $F$, let $T(A, V)$ be the set of all $u \in \mathcal{L}(E, F)$ such that $u(A) \subset V$; one takes as a fundamental system of neighborhoods of 0 in $\mathcal{L}(E, F)$ the sets $T(A, V)$, where $A$ runs through a family $\subseteq$ of subsets of $E$ and $V$ through a fundamental system of neighborhoods of 0 in $F$. It turns out that this in fact defines a locally convex topology (called $\mathfrak{S}_{\text {-topology) on }} \mathcal{L}(E, F)$, provided the sets $A \in \subseteq$ are bounded in $E$. If $E$ and $F$ are Hausdorff, $\mathcal{L}(E, F)$ is Hausdorff if the union of the sets of $\subseteq$ is dense in $E$. The family $\subseteq$ can always be supposed to consist of closed, convex, and symmetric sets, and to be such that the closed convex hull of the union of any finite number of sets of $\mathfrak{S}$ belongs to $\mathfrak{S}$. Among all S-topologies for which the union of the sets of $\widetilde{S}$ is $E$, the finest is the topology for which $\subseteq$ is the set of all bounded, convex, closed symmetric sets of $E$ (topology of bounded convergence on $\mathcal{L}(E, F)$; when $E$ and $F$ are normed spaces, it is the usual norm or "uniform" topology on $\mathcal{L}(E, F)$ ); the coarsest is the topology for which $\mathbb{S}$ is the set of all bounded convex closed finite-dimensional subsets of $E$ (topology of pointwise convergence on $\mathcal{L}(E, F)$ ).

A subset $H$ of $\mathcal{L}(E, F)$ is bounded for the $\mathfrak{S}$-topology, or $\mathfrak{S}$ bounded, if and only if for every set $A \in \subseteq$, the union of the sets $u(A)$, where $u \in H$, is bounded in $F$. This notion depends in general on the family $\varsigma_{\text {; }}$ however, if $E$ is semi-complete (see $\S 3$ ) any set which is bounded for the topology of pointwise convergence is also bounded for every S-topology $[48 ; 7]$.

In the next three sections, all spaces will be supposed to be Hausdorff locally convex spaces.

5. $t$-spaces and the Banach-Steinhaus theorem [5; 7]. Particularly important subsets of a space $\mathcal{L}(E, F)$ are the equicontinuous subsets: such a set $H$ is characterized by the property that for every neighborhood $V$ of 0 in $F$, there is a neighborhood $U$ of 0 in $E$ such that $u(U) \subset V$ for all $u \in H$. Equicontinuous subsets are $\subseteq$-bounded for every family $\mathfrak{S}$, but the converse need not hold. A locally convex 
space $E$ is called a $t$-space (French: "espace tonnelé") if for every locally convex space $F$, the subsets $H$ of $\mathcal{L}(E, F)$ which are bounded for the topology of pointwise convergence (that is, such that for every $x \in E$, the set of $u(x)$, where $u \in H$, is bounded in $F$ ) are equicontinuous (hence bounded for every S-topology). This property implies the Banach-Steinhaus theorem: for every sequence $\left(u_{n}\right)$ of continuous linear mappings of $E$ into $F$, such that $u_{n}(x)$ tends to a limit $v(x)$ for every $x \in E, v$ is a linear continuous mapping of $E$ into $F$, and $u_{n}$ converges uniformly to $v$ on every precompact subset of $E$. Moreover, if the sequence $\left(u_{n}\right)$ is such that the set of $u_{n}(x)(n=1,2, \cdots)$ is bounded in $F$ for every $x \in E$, then the same conclusion follows if convergence of $\left(u_{n}(x)\right)$ to a limit is assumed only in a dense subset of $E$, provided $F$ is semicomplete. These properties, as is well known, are among the most often used in functional analysis.

The definition of $t$-spaces can be given an equivalent formulation in which only "internal" properties of $E$ intervene. In a locally convex space $E$, a barrel is a closed, convex, symmetric, and absorbing set; closed convex symmetric neighborhoods of 0 are of course barrels. Now $E$ is a $t$-space if and only if, conversely, all barrels are neighborhoods of 0 [7].

The most important of $t$-spaces are the Baire spaces, that is, spaces in which every denumerable intersection of open dense subsets is dense [7]; this is of course the case for Banach spaces and more generally $(F)$-spaces (locally convex, metrizable, complete spaces). But there are metrizable spaces which are $t$-spaces without being Baire spaces [14]; there are also nonmetrizable complete spaces which are $t$-spaces without being Baire spaces, and complete spaces which are not $t$-spaces.

The completion of a $t$-space is a $t$-space; products and quotients of $t$-spaces are $t$-spaces [7]. However, a closed subspace of a $t$-space is not necessarily a $t$-space $[20 ; 15 ; 61]$.

6. Duality in locally convex spaces [5]. The general results on spaces $\mathcal{L}(E, F)$ of continuous linear operators apply in particular to the space $E^{\prime}=\mathcal{L}(E, R)$ of continuous linear forms (also called linear functionals); this space is called the dual (or conjugate) of $E$. The S-topologies in $E^{\prime}$ can here be described in the following simple way: for any $x \in E, x^{\prime} \in E^{\prime}$, write $\left\langle x, x^{\prime}\right\rangle=x^{\prime}(x)$; for any subset $A$ of $E$, the polar $A^{0}$ of $A$ in $E^{\prime}$ is the set of all $x^{\prime} \in E^{\prime}$ such that $\left|\left\langle x, x^{\prime}\right\rangle\right| \leqq 1$ for

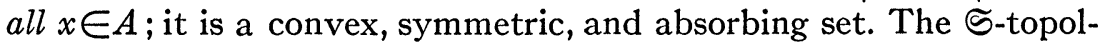
ogy on $E^{\prime}$ has a fundamental system of neighborhoods which consists of finite intersections of the polars of the sets $A \in \mathbb{S}$. The most im- 
portant S-topologies on $E^{\prime}$ are (as in general on spaces $\mathcal{L}(E, F)$ ) the topology of pointwise convergence, also called weak* topology, and denoted by $\sigma\left(E^{\prime}, E\right)$, and the topology of bounded convergence, also called strong topology, and denoted by $\beta\left(E^{\prime}, E\right.$ ) (when $E$ is a normed space, $\beta\left(E^{\prime}, E\right)$ is simply the usual norm topology on $E^{\prime}$ ).

Equicontinuous subsets of $E^{\prime}$ are those which are contained in polars $V^{0}$ of neighborhoods of 0 in $E$; these sets $V^{0}$ are weakly* compact by Tychonoff's theorem. Equicontinuous sets are strongly bounded, and strongly bounded sets are weakly* bounded; in general these three classes of sets are distinct. However:

(1) every weakly* bounded set in $E^{\prime}$ is equicontinuous if and only if $E$ is a $t$-space;

(2) if $E$ is semi-complete, every weakly* bounded set in $E^{\prime}$ is strongly bounded;

(3) in order that every strongly bounded set in $E^{\prime}$ be equicontinuous, it is necessary and sufficient that every barrel in $E$, which absorbs all bounded subsets of $E$, be a neighborhood of $0 ; E$ is then said to be a quasi-t-space; the completion of such a space is a $t$-space (see $\$ 5$ ).

From the last results, it follows easily that $E^{\prime}$ is weakly* quasicomplete (see $\S 3$ ) if $E$ is a $t$-space, and strongly quasi-complete if $E$ is a quasi-t-space (for conditions insuring completeness of $E^{\prime}$ (with the strong topology) see $\$ 9$ ).

Let $E^{\prime \prime}$ be the dual of the space $E^{\prime}$, when the latter is given the strong topology; $E^{\prime \prime}$ is called the bidual (or second conjugate) of $E$; not much is known in general about its properties (for instance, it is not known whether $E^{\prime \prime}$, with the strong topology $\beta\left(E^{\prime \prime}, E^{\prime}\right)$, is always complete, even if $E$ is a $t$-space).

For every $x \in E$, consider the linear form $x^{\prime} \rightarrow\left\langle x, x^{\prime}\right\rangle$ on $E^{\prime}$; this linear form $\tilde{x}$ is weakly* (hence also strongly) continuous, and therefore $x \rightarrow \tilde{x}$ is a linear mapping of $E$ into $E^{\prime \prime}$, which is easily seen to be 1-1. $E$ can thus be imbedded (algebraically) into $E^{\prime \prime}$; the topology $\sigma\left(E, E^{\prime}\right)$ induced on $E$ by the weak ${ }^{*}$ topology $\sigma\left(E^{\prime \prime}, E^{\prime}\right)$ is called the weak topology on $E$; it is coarser than the initial topology on that space, but bounded sets and closed convex sets are the same for both topologies [48; 9]. In general, the imbedding $x \rightarrow \tilde{x}$ is not onto; in order that $E^{\prime \prime}=E$, it is necessary and sufficient that every bounded subset of $E$ be relatively compact for the weak topology $\sigma\left(E, E^{\prime}\right) ; E$ is then said to be semi-reflexive. The topology induced on $E$ by the strong topology $\beta\left(E^{\prime \prime}, E^{\prime}\right)$ is in general distinct (and finer) than the initial topology on $E$; it is identical to it if and only if $E$ is a quasi-tspace (see above); the weak* closures (in $E^{\prime \prime}$ ) of the neighborhoods of 0 in $E$ for the initial topology constitute in that case a fundamental 
system of neighborhoods of 0 for $\beta\left(E^{\prime \prime}, E^{\prime}\right)$. In order that $E^{\prime \prime}=E$ and that the strong topology $\beta\left(E^{\prime \prime}, E^{\prime}\right)$ coincides with the initial topology on $E$, it is necessary and sufficient that $E$ be a semi-reflexive $t$-space; such spaces are called reflexive. The strong dual of a reflexive space is a reflexive space.

Let $F$ be a closed subspace of a locally convex vector space $E$; the dual $F^{\prime}$ of $F$ can be identified in a natural way with the quotient space $E^{\prime} / F^{0}$, where $F^{0}$ is the orthogonal subspace to $F$ in $E^{\prime}$, and the dual of the quotient space $E / F$ can be naturally identified with the subspace $F^{0}$ of $E^{\prime}$; moreover, the weak topologies $\sigma\left(F^{0}, E / F\right)$ and $\sigma\left(E^{\prime} / F^{0}, F\right)$ are respectively the induced and the quotient topology of $\sigma\left(E^{\prime}, E\right)$ [9]. However, when it comes to strong topologies, the corresponding results (which are true for Banach spaces) do not hold any more, even for $(F)$-spaces or Montel spaces (see $\$ 7)$. In particular, a closed subspace of a semi-reflexive space is still semi-reflexive, but a closed subspace of a reflexive space need not be reflexive, and a quotient space of a reflexive space is not even necessarily semireflexive (see, however, \$12).

7. Duality in special spaces. $(F)$-spaces (see §5) are next to Banach spaces in the applications to functional analysis, and their theory has been recently the subject of several papers $[13 ; 15]$, the deepest results being due to A. Grothendieck [20]. The dual $E^{\prime}$ of an $(F)$-space is always complete for the strong topology (see $\S 8$ ) but is never metrizable unless $E$ is a Banach space. Moreover, it is not always a $t$-space (for the strong topology) even if $E$ is separable [20]; however, when $E$ is reflexive, or when $E^{\prime}$ itself contains a denumerable dense subset for the strong topology (in which case $E$ is necessarily separable itself), $E^{\prime}$ is a $t$-space [20]. In every case, the bidual $E^{\prime \prime}$ is an $(F)$-space for the strong topology $[20 ; 15]$.

A locally convex space $E$ is called a Montel space if it is a $t$-space (\$5) and if every bounded closed subset of $E$ is compact. A Banach space cannot be a Montel space unless it is finite-dimensional; but a great many functional spaces are Montel spaces, for instance the space of holomorphic functions in a domain, with the compact-open topology, or most spaces which occur in the theory of distributions (see §12). A Montel space is reflexive, and its strong dual is again a Montel space; but a closed subspace of a Montel space need not be a Montel space [20]; and an example has been given [20] of a Montel space $E$, which is also a separable $(F)$-space, such that there is a quotient space $E / F$ of $E$ which is isomorphic to the Banach space $l^{1}$ (hence fails to be reflexive). 
8. Projective and inductive limits of spaces [4]. Let $\left(F_{\alpha}\right), \alpha \in A$, be a family of locally convex spaces, and let $E$ be a vector space, initially without any topology. Suppose first that for each $\alpha$ there is given a linear mapping $f_{\alpha}$ of $E$ into $F_{\alpha}$; then a locally convex topology can be defined on $E$ by the condition that it be the coarsest of all locally convex topologies on $E$ for which all the $f_{\alpha}$ are continuous; with that topology, $E$ is called the projective limit of the $F_{\alpha}$ (relative to the maps $f_{\alpha}$ ). If all $F_{\alpha}$ are Hausdorff spaces, $E$ is Hausdorff if and only if for every $x \neq 0$ in $E$ there is an $\alpha$ such that $f_{\alpha}(x) \neq 0$; the mapping $x \rightarrow\left(f_{\alpha}(x)\right)$ is then a topological imbedding of $E$ into the product space $\prod_{\alpha \in A} F_{\alpha}$. It is easily shown that every Hausdorff locally convex space can thus be imbedded into a product of Banach spaces. In order that a linear mapping $g$ from a locally convex space $G$ into a projective limit $E$ be continuous, it is necessary and sufficient that $f_{\alpha} \circ g$ be continuous for each $\alpha \in A$.

The family $\left(F_{\alpha}\right)$ and $E$ being as above, suppose now that for each $\alpha$ there is given a linear mapping $g_{\alpha}$ of $F_{\alpha}$ into $E$; then a locally convex topology can be defined on $E$ by the condition that it be the finest of all locally convex topologies on $E$ for which all the $g_{\alpha}$ are continuous; with that topology, $E$ is called the inductive limit of the $F_{\alpha}$ (relative to the maps $g_{\alpha}$ ). In order that a linear mapping $f$ from $E$ into a locally convex space $G$ be continuous, it is necessary and suffcient that $f \circ g_{\alpha}$ be continuous for each $\alpha \in A$.

When all the $F_{\alpha}$ are Hausdorff, no simple general condition is known which will guarantee that the inductive limit $E$ is Hausdorff; this property can, however, easily be checked for all the most important cases of inductive limits reviewed below. An inductive limit of $t$-spaces (respectively quasi-t-spaces) is a $t$-space (respectively quasi-t-space).

An obvious example of inductive limit is a quotient space $F / M$ (with only one $f_{\alpha}$, namely the natural mapping). Less obvious but quite important is the direct sum of an arbitrary family $\left(E_{\alpha}\right)_{\alpha \in A}$ of locally convex vector spaces $[29 ; 27]$ : this is the subspace $E$ of the (nontopologized) product $\prod_{\alpha \in A} E_{\alpha}$, consisting of elements having only a finite number of coordinates $x_{\alpha} \neq 0$; consider in $E$ the finite products $F_{J}=\prod_{\alpha \in J} E_{\alpha}$, each with the product topology, and the injection $g_{J}$ of $F_{J}$ in $E$; the direct sum topology on $E$ is the inductive limit of the topologies of the $F_{J}$ (relative to the injections $g_{J}$ ); we shall write $E=\sum_{\alpha \in A} E_{\alpha}$. Every inductive limit of the $E_{\alpha}$ (for some family of maps) which is the union of the images of the $E_{\alpha}$ is isomorphic to a quotient space of $\sum_{\alpha \in A} E_{\alpha}$. If all $E_{\alpha}$ are Hausdorff spaces, so is their direct sum. Bounded sets in $E$ are those which are 
contained in one of the products $F_{J}$. If all $E_{\alpha}$ are complete Hausdorff spaces, so is $E[27]$. An interesting particular case is that in which all the $E_{\alpha}$ are one-dimensional: this gives on the direct sum $E$ the finest locally convex topology.

There is a natural duality between direct sum and product [29]. More precisely, the dual of a direct $\operatorname{sum} E=\sum_{\alpha \in A} E_{\alpha}$ is the product $E^{\prime}=\prod_{\alpha \in A} E_{\alpha}^{\prime}$; the weak ${ }^{*}$ topology $\sigma\left(E^{\prime}, E\right)$ is the product of the topologies $\sigma\left(E_{\alpha}^{\prime}, E_{\alpha}\right)$, and the strong topology $\beta\left(E^{\prime}, E\right)$ is the product of the strong topologies $\beta\left(E_{\alpha}^{\prime}, E_{\alpha}\right)$. Conversely, the dual of the product $F=\prod_{\alpha \in A} E_{\alpha}$ is the direct sum $F^{\prime}=\sum_{\alpha \in A} E_{\alpha}^{\prime}$, and the strong topology $\beta\left(F^{\prime}, F\right)$ is the direct sum of the strong topologies $\beta\left(E_{\alpha}^{\prime}, E_{\alpha}\right)$ (this is not true for the weak* topologies). In order that $E$ or $F$ be semi-reflexive (respectively reflexive, or a Montel space) it is necessary and sufficient that each of the $E_{\alpha}$ be semi-reflexive (respectively reflexive, a Montel space).

9. $(L F)$-spaces, bornological spaces and the closed graph theorem. Another important instance of inductive limit is the case of a strictly increasing sequence $\left(E_{n}\right)$ of subspaces of a vector space $E$, such that $E$ is the union of the $E_{n}$; each $E_{n}$ is given a locally convex topology $\mathcal{T}_{n}$, such that $\mathcal{T}_{n}$ induces $\mathcal{G}_{n-1}$ on $E_{n-1}$. The space $E$, with the inductive limit topology $\mathcal{T}$ of the $\mathcal{T}_{n}$ (for the inclusion maps) is called the strict inductive limit of the $E_{n} ; \mathcal{T}$ induces $\mathcal{T}_{n}$ on each $E_{n}$, and if each $E_{n}$ is closed in $E_{n+1}, E_{n}$ is closed in $E$; moreover, in this last case, $E$ is Hausdorff if the $E_{n}$ are, and bounded sets in $E$ are those which are contained in some $E_{n}$ and bounded in $E_{n} ; E$ is never metrizable nor a Baire space [13]. Always in the case in which $E_{n}$ is closed in $E_{n+1}, E$ is complete (respectively semi-reflexive, reflexive, a Montel space) if and only if each $E_{n}$ has the same property [41].

The most interesting case is that in which the $E_{n}$ are $(F)$-spaces; $E$ is then called an $(L F)$-space [13]; many functional spaces are of this type, in particular, the space $(D)$ of Schwartz. Unfortunately, these spaces fail in general to possess many of the nice properties of $(F)$-spaces; for instance, a closed subspace $F$ of an $(L F)$-space $E$ is not necessarily an $(L F)$-space, nor is the quotient $E / F$ necessarily an $(F)$ or $(L F)$-space [20].

The third important example of inductive limit consists of spaces $E$ which are inductive limits of normed spaces $E_{\alpha}$ with respect to maps $g_{\alpha}$ such that $E$ is the union of the $g_{\alpha}\left(E_{\alpha}\right)$; these spaces are called bornological $[7 ; 15 ; 48 ; 61]$. Such a space can also be characterized by any of the two following properties: (a) any convex, symmetric set in $E$ which absorbs (see $\S 3$ ) all bounded subsets of $E$ is a neighbor- 
hood of 0 in $E$; (b) any linear mapping $u$ of $E$ into a locally convex space $F$, which transforms bounded sets into bounded sets, is continuous [48]. Every metrizable locally convex space is bornological [48]; every bornological space is a quasi-t-space (see $\$ 7$ ), but can fail to be a $t$-space (see §5); whether there are $t$-spaces which are not bornological is an unsolved problem, as is also the question whether the completion of a bornological space is bornological. The dual of a bornological space is strongly complete [7]. The strong dual of an $(F)$-space is bornological if and only if it is a $t$-space [20].

An inductive limit of bornological spaces is bornological. A product of bornological spaces is bornological if the set of factors has a cardinal at most equal to that of the continuum; whether the result extends to arbitrary products is an open question, equivalent to a problem of Ulam in measure theory $[48 ; 49 ; 15]$. Examples are known of bornological spaces having non-bornological closed subspaces $[20 ; 15 ; 61]$.

The notion of inductive limit is linked with the most extensive generalization which has been given thus far of the closed graph theorem of Banach $[13 ; 42 ; 24]$ : suppose $E$ is a bornological semicomplete (see $\S 4$ ) space, and $F$ is defined as the inductive limit of a sequence $\left(F_{n}\right)$ of $(F)$-spaces, relative to a sequence $\left(g_{n}\right)$ of linear mappings, such that $F$ is the union of the $g_{n}\left(F_{n}\right)$. Then, if $u$ is a linear mapping of $E$ into $F$ such that the graph of $u$ is closed in $E \times F$, $u$ is continuous. It follows from this that any linear continuous mapping of $F$ onto $E$ is an open homomorphism.

10. Spaces of bilinear functionals and tensor products. As already stressed above (see $\$ 4$ ), the study of spaces of operators, and more generally of spaces $\mathcal{L}(E, F)$, is a central theme in modern functional analysis: well known examples are the theory of rings of operators in Hilbert space, and the more recent theory of kernels developed by L. Schwartz in connection with his theory of distributions [57]. For deeper results on spaces $\mathcal{L}(E, F)$, a knowledge of their duality theory is of great value; this is the main starting point of the very recent theory of topological tensor products, which we are now going to consider.

Let $E$ and $F$ be $t$-spaces, $E^{\prime}$ and $F^{\prime}$ the duals of $E$ and $F$, and write $E_{s}, E_{s}^{\prime}$ for the spaces $E, E^{\prime}$ with the weak topologies $\sigma\left(E, E^{\prime}\right)$ and $\sigma\left(E^{\prime}, E\right)$ respectively, and similarly for $F$ and $F^{\prime}$. Every continuous linear mapping $u$ from $E$ to $F$ is also a continuous linear mapping from $E_{s}$ to $F_{s}$, and conversely, in other words, $\mathcal{L}(E, F)=\mathcal{L}\left(E_{s}, F_{s}\right)$. Now, for every $x \in E$ and every $y^{\prime} \in F^{\prime}$, consider the number $v\left(x, y^{\prime}\right)$ $=\left\langle u(x), y^{\prime}\right\rangle$; this is a bilinear functional on $E \times F^{\prime}$, and it is continu- 
ous with respect to each variable, when considered as defined over the topological space $E_{s} \times F_{s}^{\prime}$; conversely, any such separately continuous bilinear functional over $E_{s} \times F_{s}^{\prime}$ has the form $\left(x, y^{\prime}\right)$ $\rightarrow\left\langle u(x), y^{\prime}\right\rangle$, where $u$ is a well determined element of $\mathcal{L}(E, F)$; in other words, there is an algebraic isomorphism between the space $\mathcal{L}(E, F)$ and the space $\mathfrak{B}\left(E_{s}, F_{s}^{\prime}\right)$ of separately continuous bilinear functionals over $E_{s} \times F_{s}^{\prime}$.

Changing slightly our point of view, let us now consider generally the space $\mathfrak{B}(E, F)$ of bilinear separately continuous functionals over a product $E \times F$ of two locally convex spaces. We observe that this space contains, of course, the subspace $\mathcal{B}(E, F)$ of all continuous bilinear functionals (with respect to both variables), but is not generally identical with it (although it is well known that the two spaces are identical if $E$ and $F$ are $(F)$-spaces). We also observe that $\mathfrak{B}(E, F)$ and $\mathfrak{B}\left(E_{s}, F_{s}\right)$ are obviously identical, which provides the link with the preceding considerations.

Now, looking for a duality theory adapted to $\mathfrak{B}(E, F)$ (or $\mathfrak{B}(E, F)$ ) means that we are trying to determine a space such that the bilinear functionals over $E \times F$ become linear functionals over that space. In pure algebra, this has been achieved long ago by the consideration of the tensor product (or Kronecker product) $E \otimes F$ of the two spaces [2]; this is defined as the set of all formal (finite) linear combinations $\sum_{i} x_{i} \otimes y_{i}\left(x_{i} \in E, y_{i} \in F\right)$, where identifications have been performed, such that $\left(x+x^{\prime}\right) \otimes\left(y+y^{\prime}\right)$ is identified with $x \otimes y+x^{\prime} \otimes y+x \otimes y^{\prime}$ $+x^{\prime} \otimes y^{\prime}$, and $(\alpha x) \otimes(\beta y)$ with $\alpha \beta(x \otimes y)$. Linear forms on that space are exactly in one-to-one correspondence with all bilinear forms on $E \times F$, the correspondence associating to the linear form $u$ the bilinear form $(x, y) \rightarrow u(x \otimes y)$. When $E$ and $F$ are locally convex spaces, we can still consider the algebraic tensor product $E \otimes F$, and $\mathfrak{B}(E, F)$ (or $\mathcal{B}(E, F)$ ) can then be identified with a space of linear forms on $E \otimes F$; but we want to define $E \otimes F$ as a topological vector space in such a way that the elements of $\mathfrak{B}(E, F)$ (or $\mathcal{B}(E, F)$ ) will become continuous over $E \otimes F$. This problem was first successfully attacked by R. Schatten when $E$ and $F$ are Banach spaces [55]; it has now been extensively treated in the general case by A. Grothendieck $[23 ; 24]$. It turns out that, even in the case of Banach spaces, there are several "natural" solutions.

Let us suppose, for simplicity's sake, that $E$ and $F$ are complete $t$-spaces. The spaces $E \otimes F$ and $B(E, F)$ are put in a natural duality by the "scalar product" $\langle x \otimes y, u\rangle=u(x, y)$ as seen above. If we recall that, in the dual of a locally convex space $G$, polars of the neighborhoods of 0 in $G$ are equicontinuous sets (see $\$ 6$ ), we have a 
natural way of defining neighborhoods of 0 in $E \otimes F$ : for each equicontinuous set $M$ of continuous bilinear functionals, consider the set $M^{0}$ in $E \otimes F$ consisting of all $z=\sum_{i} x_{i} \otimes y_{i}$ such that $|\langle z, u\rangle|$ $=\left|\sum_{i} u\left(x_{i}, y_{i}\right)\right| \leqq 1$ for all $u \in M$. A fundamental system of neighborhoods of 0 in $E \otimes F$ will then be taken as consisting of all sets of the form $M^{0}$. There are two equivalent definitions: (a) take a convex neighborhood $V$ of 0 in $E$, a convex neighborhood $W$ of 0 in $F$, and consider the convex hull $\Gamma(V \otimes W)$ of all elements $x \otimes y$, where $x \in V$ and $y \in W$; these sets form a fundamental system of neighborhoods of 0 ; (b) equivalently, take a semi-norm $\alpha$ on $E$, a semi-norm $\beta$ on $F$, and define, for $z \in E \otimes F$,

$$
\gamma(z)=\text { g.l.b. }\left(\sum_{i} \alpha\left(x_{i}\right) \beta\left(y_{i}\right)\right)
$$

the g.l.b. being taken over all possible representations of $z$ in the form $\sum_{i} x_{i} \otimes y_{i}$; then the semi-norms $\gamma$ define the topology of $E \otimes F$. For that topology, linear continuous functionals on $E \otimes F$ are exactly identified with the bilinear continuous functionals on $E \times F$; more generally, for any locally convex space $H$, the space $\mathcal{L}(E \otimes F, H)$ is identified with the space of continuous bilinear mappings of $E \times F$ into $H$.

The preceding operations, however, still give in general "too small" a vector space for the tensor product. This is best understood when one of the spaces $E, F$ is a space of numerical functions over some set $S$; it is then easily verified that the space $E \otimes F$ (without topology) can be identified with the set of mappings $t \rightarrow \sum_{i} x_{i}(t) y_{i}$ (finite sum), where $x_{i} \in E$ and $y_{i} \in F$; these are mappings of finite rank, and it is clear that interesting results will be obtained only if we enlarge that functional space by adding to it the "limits" of its elements in some sense. This leads to the final step in the definition of the tensor product: we consider the space $E \otimes F$ with the topology defined above and take its completion $E \otimes_{1} F$, which is then called the projective tensor product of $E$ and $F$.

Similar definitions may be given when $\mathcal{B}(E, F)$ is replaced by $\mathfrak{B}(E, F)$; we take here as sets $M \subset \mathfrak{B}(E, F)$ those which are separately equicontinuous (that is, for every $x \in E$, the set of linear functionals $y \rightarrow u(x, y)$ is equicontinuous when $u$ runs through $M$, and the same condition holds when $E$ and $F$ are exchanged). The polar sets $M^{0}$ then define a new topology on $E \otimes F$, and the completion of $E \otimes F$ for that topology is a second tensor product, written $E \otimes_{0} F$ and called the inductive tensor product. It is identical to $E \otimes_{1} F$ when both $E$ and $F$ are $(F)$-spaces. Continuous linear mappings of $E \otimes_{0} F$ into a com- 
plete locally convex space $H$ are here identified with the separately continuous bilinear mappings of $E \times F$ into $H$.

A third "natural" topology is finally defined in the following way. Let us consider the duals $E^{\prime}$ and $F^{\prime}$ of $E$ and $F$, and their tensor product $E^{\prime} \otimes F^{\prime}$; this space (without topology) can be identified in a natural way with a subspace of $\mathcal{B}(E, F)$, by associating to the tensor product $x^{\prime} \otimes y^{\prime}$ the bilinear form $(x, y) \rightarrow\left\langle x, x^{\prime}\right\rangle\left\langle y, y^{\prime}\right\rangle$ (product in the classical sense of $x^{\prime}$ and $\left.y^{\prime}\right)$. We can now take as sets $M$ in the general method outlined above the products $P \otimes Q$ (set of all $x^{\prime} \otimes y^{\prime}$, where $x^{\prime} \in P, y^{\prime} \in Q$ ), where $P$ and $Q$ are equicontinuous subsets of $E^{\prime}$ and $F^{\prime}$ respectively. The completion of $E \otimes F$ under this topology is called the biprojective tensor product of $E$ and $F$, and written $E \otimes_{2} F$.

Of these three topologies on $E \otimes F$, the biprojective is the coarsest, the inductive the finest, and the projective is between these two extremes. Hence the "largest" tensor product is the biprojective one, and the "smallest" the inductive one, the projective tensor product being "intermediate."

11. Properties of the tensor products [24]. We shall only be concerned with the projective and biprojective tensor products. A "concrete" interpretation can be given of these products in several important cases. For instance, if $E$ and $F$ are $(F)$-spaces, then $E \otimes_{1} F$ and $E \otimes_{2} F$ are $(F)$-spaces; the elements of $E \otimes_{1} F$ can be described as sums of convergent series $\sum_{k} \lambda_{k} x_{k} \otimes y_{k}$, where $\sum_{k=0}^{\infty}\left|\lambda_{k}\right|$ is finite, and the sequences $\left(x_{k}\right)$ and $\left(y_{k}\right)$ tend to 0 in $E$ and $F$ respectively. The space $l^{1} \otimes_{1} E$ can be identified with the space of absolutely convergent series in $E$ (that is, series $\sum_{n=0}^{\infty} x_{n}$ such that for every seminorm $p$ on $E$, the series $\sum_{n=0}^{\infty} p\left(x_{n}\right)$ is absolutely convergent), whereas the space $l^{1} \otimes_{2} E$ can be identified with the space of all unconditionally convergent series in $E$. Similarly, if $C(K)$ is the space of real continuous functions on a compact space $K, C(K) \otimes_{2} E$ can be identified with the space of continuous mappings from $K$ to $E$, with the topology of uniform convergence.

Other important interpretations of tensor products stem from the initial remark of $\S 10$. Every element $\sum_{i} x_{i}^{\prime} \otimes y_{i}$ of the (algebraic) tensor product $E^{\prime} \otimes F$ can be identified with the linear mapping $x \rightarrow \sum_{i}\left\langle x, x_{i}^{\prime}\right\rangle y_{i}$ of $E$ into $F$; in other words, $E^{\prime} \otimes F$ can be considered as the space of all linear continuous mappings of finite rank of $E$ into $F$. The tensor products $E^{\prime} \otimes_{1} F$ and $E^{\prime} \otimes_{2} F$ (where $E^{\prime}$ is given its strong topology) will, in the most important cases, be identified with spaces of linear mappings of $E$ into $F$ which are "limits" in some sense of mappings of finite rank. For instance, if $E$ is a reflexive Banach space with a basis, $E^{\prime} \otimes_{2} E$ will be identified with the space of com- 
pletely continuous mappings of $E$ into itself, whereas, in general, $E^{\prime} \otimes_{1} E$ will be a proper subspace of that space (for instance, when $E$ is a Hilbert space, a completely continuous hermitian operator will belong to $E^{\prime} \otimes_{1} E$ only if the series of its eigenvalues is absolutely convergent $[14 ; 55])$. The dual of $E^{\prime} \otimes_{2} E$ is then identified with $E \otimes_{1} E^{\prime}$; hence, its bidual with $\mathcal{B}\left(E^{\prime}, E\right)$ [14].

This shows that in general the dual of $E \otimes_{2} F$ will not be equal to $\mathscr{B}(E, F)$. It is much smaller, and can be determined directly from the duals $E^{\prime}, F^{\prime}$ in the following explicit way. The (algebraic) spaces $E \otimes F$ and $E^{\prime} \otimes F^{\prime}$ are in a natural duality, defined by $\left\langle x \otimes y, x^{\prime} \otimes y^{\prime}\right\rangle$ $=\left\langle x, x^{\prime}\right\rangle\left\langle y, y^{\prime}\right\rangle$; the topology of $E \otimes_{2} F$ is such that $x^{\prime} \otimes y^{\prime}$ can be extended by continuity to a continuous linear functional on $E \otimes_{2} F$. Now, all continuous linear functionals on $E \otimes_{2} F$ can be obtained from these "elementary" ones by a process of integration: more precisely, any such functional can be written

$$
u \rightarrow \int\left\langle u, x^{\prime} \otimes y^{\prime}\right\rangle d \mu\left(x^{\prime}, y^{\prime}\right)
$$

where $\mu$ is a positive Radon measure defined on a product $P \times Q$ of an equicontinuous (hence compact) subset $P$ of $E^{\prime}$ and an equicontinuous subset $Q$ of $F^{\prime}$. This, applied to $E^{\prime} \otimes_{2} F$, leads to important classes of linear mappings of $E$ into $F$, which have many useful properties.

12. Nuclear spaces [24]. Comparison of the two tensor products $E \otimes_{1} F$ and $E \otimes_{2} F$ has led A. Grothendieck to the investigation of a new class of locally convex spaces, namely those spaces $E$ for which $E \otimes_{1} F$ and $E \otimes_{2} F$ are (topologically) identical for every locally convex space $F$. Such spaces are called nuclear; they possess many remarkable properties, which make them closer to finite-dimensional spaces than any other known category of infinite-dimensional spaces.

A space is nuclear if and only if its completion is nuclear; we shall therefore consider only complete nuclear spaces. In such a space, a bounded set is always relatively compact, hence the space is always semi-reflexive ( $\$ 6)$, but it need not be a $t$-space; if it is a $t$-space, it is of course a Montel space; moreover, if it is an $(F)$-space, its (strong) dual is also a nuclear space. A closed subspace $F$ of a nuclear space $E$ is nuclear and so is the quotient space $E / F$. Any projective limit of nuclear spaces $(\$ 8)$ is nuclear; so is the inductive limit of a sequence of nuclear spaces.

If both $E$ and $F$ are (complete) nuclear spaces, so is their tensor product $E \otimes_{1} F=E \otimes_{2} F$ : moreover, $E \otimes_{1} F$ can be identified with the 
whole space $\mathfrak{B}\left(E^{\prime}, F^{\prime}\right)$ of separately continuous bilinear functionals on $E^{\prime} \times F^{\prime}$. As seen above, the dual of $E \otimes_{2} F$ is then $\mathcal{B}(E, F)$ (hence, all continuous bilinear functionals on $E \times F$ have the "integral" representation given at the end of \$11); moreover, if $E$ and $F$ are $(F)$-spaces, $\mathcal{B}(E, F)$ can be identified (topologically) with the tensor product $E^{\prime} \otimes_{1} F^{\prime}$ of the (strong) duals. In the same case, the space $\mathcal{L}(E, F)$, with the topology of bounded convergence ( $\$ 4)$ is nuclear, and its strong dual is also nuclear.

The $(F)$-spaces which are nuclear spaces can be characterized by the following property: they are the only $(F)$-spaces in which every unconditionally convergent series is also absolutely convergent: for Banach spaces, this gives of course, in particular, an entirely new proof of the Dvoretzky-Rogers theorem [16].

The importance of nuclear spaces lies chiefly in the fact that most spaces which occur in the theory of distributions, or in the theory of analytic functions, are nuclear spaces (for instance, the spaces $(\mathcal{D}),\left(\mathcal{D}^{\prime}\right),(\mathcal{E}),\left(\mathcal{E}^{\prime}\right)$ of Schwartz $[56 ; 57]$, as well as the space $\mathfrak{H C}(G)$ of holomorphic functions in a domain, with the topology of compact convergence (compact-open topology) $[58 ; 8 ; 45 ; 46 ; 25]$ ). The simple properties of nuclear spaces will undoubtedly prove very useful in later developments of these theories.

\section{BiBLIOGRAPHY}

1. R. Arens, Duality in linear spaces, Duke Math. J. vol. 14 (1947) pp. 787-794.

2. N. Bourbaki, Eléments de mathematique. VII. Part 1, Les structures fondamentales de l'analyse. Book II. Algèbre. Chapter III: Algèbre multilinéaire, Actualités Scientifiques et Industrielles, no. 1044, Paris, Hermann, 1948, 2+157+2 pp.

3. - Éléments de mathématique. X. Part 1. Les structures fondamentales de l'analyse. Book III. Topologie gênérale. Chapter X. Espaces fonctionnels; dictionnaire, Actualités Scientifiques et Industrielles, no. 1084, Paris, Hermann, 1949. ii +101 pp.

4. - Eléments de mathêmatique. XV. Part 1. Les structures fondamentales de l'analyse. Book V. Espaces vectoriels topologiques. Chapter I. Espaces vectoriels topologiques sur un corps valué. Chapter II. Ensembles convexes et espaces localement convexes, Actualités Scientifiques et Industrielles, no. 1189, Paris, Hermann, 1953, 2+124 $+4 \mathrm{pp}$.

5. - Espaces vectoriels topologiques, Chapters III-V. (To be published shortly.)

6. - Eléments de mathêmatique. XIII. Part 1. Les structures fondamentales de l'analyse. Book VI. Intégration. Chapter I. Inégalités de convexité. Chapter II. Espaces de Riesz. Chapter III. Mesures sur les espaces localement compacts. Chapter IV. Prolongement d'une mesure, espaces $L^{p}$, Actualités Scientifiques et Industrielles, no. 1175, Paris, Hermann, 1952.

7. - Sur certains espaces vectoriels topologiques, Ann. Inst. Fourier Grenoble vol. 2 (1950) pp. 5-16 (1951).

8. C. da Silva Dias, Espą̧os vectorias topologicos e sua applica̧̧ão na teoria dos espaços funcionais analtiticos (Topological vector spaces and their applications in the 
theory of analytic functional spaces), Thesis, University of São Paulo, 1951, 2+66 pp.

9. J. Dieudonné, La dualitê dans les espaces vectoriels topologiques, Ann. École Norm. (3) vol. 59 (1942) pp. 107-139.

10. - Natural homomorphisms in Banach spaces, Proc. Amer. Math. Soc. vol. 1 (1950) pp. 54-59.

11. - Sur les espaces de Köthe, J. Analyse Math. vol. 1 (1951) pp. 81-115.

12. J. Dieudonné and A. P. Gomes, Sur certains espaces vectoriels topologiques, C. R. Acad. Sci. Paris vol. 230 (1950) pp. 1129-1130.

13. J. Dieudonné and L. Schwartz, La dualitê dans les espaces $(\mathcal{F})$ et $(\mathcal{L} \mathcal{F})$, Ann. Inst. Fourier Grenoble vol. 1 (1949) pp. 61-101 (1950).

14. J. Dixmier, Les fonctionnelles linéaires sur l'ensemble des opérateurs bornés d'un espace de Hilbert, Ann. of Math. (2) vol. 51 (1950) pp. 387-408.

15. W. F. Donoghue and K. T. Smith, On the symmetry and bounded closure of locally convex spaces, Trans. Amer. Math. Soc. vol. 73 (1952) pp. 321-344.

16. A. Dvoretzky and C. A. Rogers, Absolute and unconditional convergence in normed linear spaces, Proc. Nat. Acad. Sci. U. S. A. vol. 36 (1950) pp. 192-197.

17. A. Grothendieck, Sur la complétion du dual d'un espace vectoriel localement convexe, C. R. Acad. Sci. Paris vol. 230 (1950) pp. 605-606.

18. - Quelques résultats relatifs a la dualité dans les espaces ( $\mathcal{F}), \mathrm{C} . \mathrm{R}$. Acad. Sci. Paris vol. 230 (1950) pp. 1561-1563.

19. - Critères gênéraux de compacitê dans les espaces vectoriels localement convexes. Pathologie des espaces ( $\mathcal{L} 7$ ), C. R. Acad. Sci. Paris vol. 231 (1950) pp. 940-941.

20. - Sur les espaces $(\mathcal{F})$ et $(\mathcal{D} \mathcal{F})$. (To be published in Summa Bras. Math.)

21. - Quelques résultats sur les espaces vectoriels topologiques, C. R. Acad. Sci. Paris vol. 233 (1951) pp. 839-841.

22. - Sur les applications linéaires faiblement compactes d'espaces du type $C(K)$, Canadian Journal of Mathematics vol. 5 (1953) pp. 129-173.

23. - Sur une notion de produit tensoriel topologique d'espaces vectoriels topologiques, et une classe remarquable d'espaces vectoriels liée à cette notion, $\mathrm{C}$. R. Acad. Sci. Paris vol. 233 (1951) pp. 1556-1558.

24. - Produits tensoriels topologiques et espaces nucléaires. (To be published in the Memoirs of the American Mathematical Society.)

25. - Sur certains espaces de fonctions holomorphes. (To be published in J. Reine Angew. Math.)

26. E. Hagemann, Das Reziprokentheorem in beliebigen linearen Koordinatenräumen, Math. Ann. vol. 114 (1937) pp. 126-143. 954.

27. S. Kaplan, Cartesian products of reals, Amer. J. Math. vol. 74 (1952) pp. 936-

28. M. Katětov, Zur Theorie der topologischen Vektorräume, Acad. Tchèque Sci. Bull. Int. Cl. Sci. Math. Nat. vol. 44 (1943) pp. 599-605.

29. - On convex topological linear spaces, Acta Fac. Nat. Univ. Carol. Prague, no. 181, 1948, $20 \mathrm{pp}$.

30. V. L. Klee, Jr., Convex sets in linear spaces, Duke Math. J. vol. 18 (1951) pp. 443-466 and 875-883.

31. A. Kolmogoroff, Zur Normierbarkeit eines allgemeinen topologischen linearen Raumes, Studia Math. vol. 5 (1935) pp. 29-33.

32. G. Köthe and $\mathrm{O}$. Toeplitz, Lineare Räume mit unendlich vielen Koordinaten und Ringe unendlicher Matrizen, J. Reine Angew. Math. vol. 171 (1934) pp. 193-226.

33. G. Köthe, Die konvergenzfreien linearen Räume abzahlbarer Stufe, Math. Ann. vol. 111 (1935) pp. 229-258. 
34. — Die Teilräume eines linearen Koordinatenraumes, Math. Ann. vol. 114 (1937) pp. 99-125.

35. - Lösbarkeitsbedingungen für Gleichungen mit unendlich vielen Unbekannten, J. Reine Angew. Math. vol. 178 (1938) pp. 193-213.

36. —_, Das Reziprokentheorem fur zeilenabsolute Matrizen, Monatsh. Math. Phys. vol. 47 (1939) pp. 224-233.

37. - Erweiterung von Linearfunktionen in linearen Räumen, Math. Ann. vol. 116 (1939) pp. 719-732.

38. - Die Quotientenräume eines linearen vollkommenen Räumes, Math. Zeit. vol. 51 (1947) pp. 17-35.

39. - Die Stufenräume, eine einfache Klasse linearer vollkommener Räume, Math. Zeit. vol. 51 (1948) pp. 317-345.

40. - Eine axiomatische Kennzeichnung der linearen Räume vom Typus $\omega$, Math. Ann. vol. 120 (1949) pp. 634-649.

41. - Über die Vollständigkeit einer Klasse lokalkonvexer Räume, Math. Zeit. vol. 52 (1950) pp. 627-630.

42. —- Über zwei Sätze von Banach, Math. Zeit. vol. 53 (1950) pp. 203-209.

43. - Neubegrïndung der Theorie der vollkommenen Räume, Math. Nachr. vol. 4 (1951) pp. 70-80.

44. - Die verschiedenen Reziproken einer unendlichen Matrix, Monatsh. Math. Phys. vol. 55 (1951) pp. 153-156.

45. — Dualität in der Funktionentheorie, J. Reine Angew. Math. vol. 191 (1953) pp. 29-49.

46. - Die Randverteilungen analytischer Funktionen, Math. Zeit. vol. 57 (1952) pp. 13-33.

47. G. Mackey, On infinite-dimensional linear spaces, Trans. Amer. Math. Soc. vol. 57 (1945) pp. 155-207.

48. - On convex topological linear spaces, Trans. Amer. Math. Soc. vol. 60 (1946) pp. 519-537.

49. - Equivalence of a problem of measure theory to a problem in the theory of vector lattices, Bull. Amer. Math. Soc. vol. 50 (1944) pp. 719-722.

50. F. Menn, Die konvergenzfreien Räume endlicher Stufe und die dazugehörigen Matrizenringe, Dissertation, Münster, 1934.

51. E. Michael, Transformations from a linear space with weak topology, Proc. Amer. Math. Soc. vol. 3 (1952) pp. 671-676.

52. - Locally multiplicatively-convex topological algebras, Memoirs of the American Mathematical Society vol. 11 (1952).

53. H. Nakano, Topology and linear topological spaces, Tokyo Math. Book Series, vol. $3,1951$.

54. J. von Neumann, On complete topological spaces, Trans. Amer. Math. Soc. vol. 37 (1935) pp. 1-20.

55. R. Schatten, $A$ theory of cross-spaces, Annals of Mathematics Studies, no. 27, Princeton University Press, 1950.

56. L. Schwartz, Théorie des distributions, vol. I, Actualités Scientifiques et Industrielles, no. $1091=$ Publ. Inst. Math. Univ. Strasbourg 9, Paris, Hermann, 1950, 148 pp.; vol. II, Actualités Scientifiques et Industrielles no. $1122=$ Publ. Inst. Math. Univ. Strasbourg 10, Paris, Hermann, 1951. 169 pp.

57. - Theorie des noyaux, Proceedings of the International Congress of Mathematicians, Cambridge, Massachusetts, 1950, Providence, American Mathematical Society, 1952, vol. 1, pp. 220-230. 
58. J. Sebastião e Silva, Analytic functions and functional analysis, Port. Math. vol. 9 (1950) pp. 1-130.

59. G. Sirvint, Espace de fonctionnelles linéaires, C. R. (Doklady) Acad. Sci. URSS. vol. 26 (1940) pp. 123-126.

60. V. Smulian, Über lineare topologische Räume, Rec. Math. (Mat. Sbornik) N. S. vol. 7 (1940) pp. 425-448.

61. O. Takenouchi, Sur les espaces linéaires localement convexes, Math. J. Okayama Univ. vol. 2 (1952) pp. 57-84.

62. O. Toeplitz, Die linearen vollkommenen, Räume der Funktionentheorie, Comment. Math. Helv. vol. 23 (1949) pp. 222-242.

63. J. V. Wehausen, Transformations in linear topological spaces, Duke Math. J. vol. 4 (1938) pp. 157-169.

UNIVERSITY OF MICHIGAN 